\title{
Central Nervous System thrombotic microangiopathy in COVID-19 patients: an intriguing hypothesis?
}

\author{
Cristina Agostinis \\ ASST Papa Giovanni XXIII \\ Angela Napolitano \\ ASST Papa Giovanni XXIII \\ Mariangela Cava \\ ASST Papa Giovanni XXIII \\ Emilio Giazzi \\ ASST Papa Giovanni XXIII \\ Andrea Gianatti \\ ASST Papa Giovanni XXIII \\ Paolo Gritti \\ ASST Papa Giovanni XXIII \\ Nicola Rifino \\ ASST Papa Giovanni XXIII \\ Luca Giovanni Longhi \\ ASST Papa Giovanni XXIII \\ Ferdinando Luca Lorini \\ ASST Papa Giovanni XXIII \\ Maria Sessa \\ ASST Papa Giovanni XXIII \\ Simonetta Gerevini ( $\nabla$ sgerevini@asst-pg23.it ) \\ ASST Papa Giovanni XXIII
}

\section{Research Article}

Keywords: Coronavirus, Magnetic resonance imaging, Central nervous system, Inflammation, Endothelium vascular

Posted Date: October 6th, 2020

DOl: https://doi.org/10.21203/rs.3.rs-88430/v1

License: () (1) This work is licensed under a Creative Commons Attribution 4.0 International License. Read Full License 


\section{Abstract}

Objectives: Neurological manifestations of COVID-19 disease are being increasingly recognized. A growing number of studies has been showing CNS abnormalities on brain imaging.

Purpose is to describe brain imaging findings of a population of COVID-19 patients with neurological manifestations and peculiar abnormalities on susceptibility weighted imaging (SWI) sequences.

Methods: we retrospectively evaluated imaging data from 50 patients affected by SARS-CoV-2 infection, who underwent a brain MRI because of neurological symptoms between March and June 2020.

We focused on those presenting with abnormalities on brain MRI on SWI (positive MRI, P-MRI). We also carried out comparative investigations using patients without SWI abnormalities (negative MRI, N-MRI) as a control group. Non-parametric tests were used. A p-value $<0.05$ was considered significant.

Results: From 50 patients presenting with neurological symptoms, 10 patients showed brain abnormalities on SWI (P-MRI). Mean age in P-MRI group was $63 \pm 12$ years; 7 were men. Six patients were admitted to Intensive Care Units (ICUs) and needed invasive ventilation support. P-MRI subjects showed lymphopenia and significantly higher levels of inflammatory markers such as CRP, IL-6, and fibrinogen. No significant differences were found in the coagulation profile.

MRI showed diffuse SWI hypointense lesions mostly in occipital and temporal lobes, predominantly located at the grey-white matter junction. Genu and splenium of corpus callosum were involved in 8 of 10 patients. No restricted diffusion or enhancement was associated to SWI lesions.

Conclusions: SWI abnormalities in patients with COVID-19 with neurological symptoms may reflect microvascular endothelial damage in the setting of a pro-inflammatory state.

\section{Key Points}

- COVID-19 patients with neurological symptoms may present with peculiar abnormalities on

susceptibility weighted imaging (SWI) sequence.

- Patients showing cerebral microbleeds present with altered mental status and/or confusion.

- SWI microbleeds in COVID-19 patients may reflect microvascular endothelial damage in the setting

of a pro-inflammatory state (affecting both white and gray matter being visible at the perivascular

space)

\section{Introduction}

The novel coronavirus SARS-CoV-2 has caused an outbreak of severe pneumonia and related coronavirus diseases (COVID-19) in China, rapidly spreading worldwide. Italy and especially Lombardy have been involved.

Although the predominant clinical presentation is a respiratory disease, there is a growing evidence from literature of CNS involvement during SARS-Cov-2 infection. The most common symptoms include stroke (especially aphasia), headache, psychiatric disorders, and hyposmia/ageusia.[1] [2] [3]

The binding of SARS-Cov-2 to angiotensin converting enzyme 2 (ACE2) is a critical step in the pathophysiology of clinical manifestations in patients with COVID-19 [4], as it triggers the formation of a cytokine storm, with marked elevation in the levels of interleukin-1, interleukin-6, and tumor necrosis factor [5]. High levels of these cytokines increase vascular permeability, edema, and widespread inflammation with consequent multiorgan damage [6]. ACE2 receptor is widely expressed on human cells in multiple organs, including blood vessels and the brain [7].

It is well documented that SARS-CoV-2 can cause damage to endothelial cells in the lungs, the heart, and the kidneys, activating inflammatory and thrombotic pathways. [8] Endothelial cell infection or monocyte activation, upregulation of tissue factors, and the release of microparticles, which activate the thrombotic pathway and cause microangiopathy, might occur for SARS-CoV-2 as for other viruses. [9]

Thrombocytopenia with elevated D-dimer and C-reactive protein in severe COVID-19 and the high prevalence of thrombotic lesions in these patients are consistent with a virus-associated microangiopathic process. Endothelial dysfunction can potentially lead to microvascular and macrovascular complications in the brain, as described so far [10]

On the other hand, current literature is limited regarding neuroimaging findings of patients with COVID-19.

In this case series we report 10 patients with neurological symptoms during COVID 19 infection presenting with peculiar brain lesions being clearly evident on SWI sequence.

\section{Materials And Methods}




\section{Study design and patient selection}

We retrospectively evaluated data from 50 patients affected by SARS-CoV-2 infection, who underwent a brain MRI because of neurological symptoms between March and June 2020 at our institution.

We focused the present analysis on all patients presenting with abnormalities on brain MRI on susceptibility weighted imaging (SWI), excluding patients with large ischemic infarction with micro-hemorrhagic foci documented both on CT and MRI.

Furthermore, we carried out comparative investigations with the overall group of patients.

5 patients $\left(n^{\circ} 2,3,4,5,6\right)$ had monthly follow up. Patient $n^{\circ} 2,3,5,6$ had 3 MRI, Patient $n^{\circ} 4$ had 2 MRI.

\section{COVID-19 diagnosis}

COVID-19 diagnosis was confirmed: 1) by real-time reverse-transcriptase polymerase-chain-reaction (RT-PCR) on nasopharyngeal specimens; or 2) by RTPCR on bronchoalveolar lavage (BAL) obtained by bronchoscopy in case of high clinical suspicion of SARS-CoV-2 infection and negative test results on at least two nasopharyngeal swabs performed at least 24 hours apart; or 3 ) in the presence of characteristic radiological interstitial pneumonia associated with typical symptoms (fever, dry cough, dyspnea), even with negative RT-PCR [11].

\section{Clinical and Laboratory data.}

Clinical and laboratory data were extracted from the patients' electronic medical records in the Hospital Information System. We evaluated leukocytes, lymphocytes, CRP, LDH, fibrinogen values, D-dimer, IL-6 values, focusing on the worst value, and platelet counts (PLT) as maximum and minimum values. Worst ventilation parameters (FiO2, $\mathrm{PaO} 2, \mathrm{PaO} 2 / \mathrm{FiO} 2)$ were recorded.

\section{Brain MR}

All patients with clinical neurological symptoms were studied on a 3 T MRI scanner (GE MR $750 \mathrm{~W}$ ) with a dedicated MRI protocol, that included Axial and coronal T2 Propeller HR 3mm, Ax TOF 3D Fs, Sag Cube Flair Isotropic, Ax T1 Memp 3mm, Ax DTI 2mm 64 Direction, Ax DWI 2 mm All Brain Coverage and 3D T1- weighted spin-echo AX Swan HR Before \& After Contrast administration. All anonymized images were reviewed by two experienced neuroradiologists $(S G, C A)$ and three youngest colleagues (EG, AN, MC).

\section{Statistical analysis}

Age (normal distribution) was presented as age and standard deviation. The remaining data do not have a normal distribution and are presented as median and 25th - 75th percentiles. Comparisons between patients with positive MRI and negative MRI were done using the Mann Whitney test. The graph-pad Prism software was used for statistical analysis. A p-value $<0.05$ was considered statistically significant.

Collection and scientific use of the patient's data are part of a larger observational study protocol approved by the hospital ethics committee (reg 2020144).

\section{Results}

From a total number of 50 patients with COVID-19 who underwent brain MRI in the aforementioned period, 10 patients showed brain abnormalities on SWI (from now Positive MRI group, P-MRI).

The average age of the patients in P-MRI group was $63 \pm 12$ (AVG \pm SD) years; 7 were men.

6 patients were admitted to Intensive Care Units (ICUs) and needed invasive respiratory support by orotracheal intubation, lasting for a median of 10 days (range 6-26 days).

4 patients were hospitalized in a medicine department and were treated with non-invasive methods application for respiratory support (NIV/CPAP).

The neurologic symptoms were altered mental status $(8 / 10=80 \%)$, associated to a lesser extent with ischemic stroke $(10 \%)$, hemorrhagic stroke $(20 \%)$, epileptic seizure (10\%), movement disorders (10\%) or peripheral neuropathy $(20 \%)$.

9 patients survived, only one patient presenting with altered mental status had a fatal outcome.

The time elapsed between admission and the onset of neurological symptoms for which MRI was performed had a median of 9 days (25-75\% 3-23 days).

Extensive demographic and clinical findings of P-MRI patients are listed in Table 1.

4 patients underwent a lumbar puncture, and all of them had increased markers of inflammation (high white blood cell count, and/or high proteinorrachia, and/or elevated immunoglobulin G); only one patient demonstrated the presence of SARS-CoV 2 with a RT-PCR cerebrospinal fluid analysis. 
All patients underwent non-contrast enhanced chest CT scan with highly suggestive findings of COVID-19 interstitial pneumonia; in $1 / 5$ patients who performed contrast medium Chest CT scan, pulmonary thrombo-embolism was detected.

As compared with patients without documented lesions at brain MRI (Negative MRI, N-MRI, 40 pts), P-MRI subjects showed lymphopenia and higher inflammatory levels of markers such as CRP, IL-6, and fibrinogen. No significant differences were found in the coagulation profile as observed.

Furthermore, the two groups appeared absolutely identical from the respiratory and oxygen-therapy point of view, thus being effectively comparable for the other parameters (Table 2).

In all P-MRI patients brain MRI demonstrated numerous diffuse hypointense lesions on SWI in every lobe, mostly the occipital, temporal, frontal and parietal lobes, bilaterally, being occipital and temporal the prevalent location (see Table 3, Fig1). Lesions were predominantly located at the grey/white matter junction in the subarachnoid space or in the perivascular spaces, that in some cases were dilated, with both infratentorial and supratentorial distribution. Genu and splenium of corpus callosum were involved in 8 of 10 patients. Other involved regions were internal capsule, thalami, centra semiovalia and brainstem, with a predilection for the pons (Fig 2). There was no associated cerebral edema. Hypointense lesions can be "dot like" or linear. No lesion was clearly evident in the parenchyma. These hypointense lesions were clearly evident on SWI sequences, while not completely or even partially evident on Gradient Echo sequence (Fig 3), nor associated to focal or diffuse hyperdensity on CT scan (except for one case, pt ${ }^{\circ} 5$, who also showed subarachnoid hemorrhage). No hyperintense parenchymal lesions, neither focal or extensive restriction on DWI or enhancement was associated to focal hypointense SWI lesions; in 4 cases meningeal enhancement was present.

At monthly follow up imaging (1-2-3 months) all of 5 patients presented with an apparent worsening of MRI findings, with a more evident and "dark" hypointensity on SWI sequences, while clinically stable. No modifications on CT scan or T2 images were found.

\section{Discussion}

Neurological complications following COVID-19 infection are not uncommon[1]. Several putative mechanisms for CNS involvement have been suggested. [12]

Vascular changes affecting multiple organs are among the distinctive features of COVID-19. The exact underlying pathophysiological mechanism remains mostly unknown.

In COVID-19 pneumonia, the extensive microvascular damage seems to be related to a macrophage activation syndrome (MAS)-like mechanism [13] which differs from disseminated intravascular coagulopathy (DIC) and induces a coagulopathic cascade with subsequent local microthrombosis and microbleeding in the small pulmonary vessels.

A similar immune-mediated microvascular damage could be responsible for CNS manifestations [12], but supporting data are still scarce.

In this case series we presented patients with MRI signs of brain microvascular injury, characterized by small SWI hypointensities with a peculiar distribution.

Corpus callosum is one of the more frequent location of the lesions.

Similar imaging findings are reported in severe acute respiratory distress syndromes[14], including high altitude cerebral edema (HACE). [15] Interestingly, in these published cases, microbleeds in the corpus callosum are associated with a restriction on diffusion-weighted sequences, as for cytotoxic edema. The localization of the lesions is very similar to what observed in our series; however, in none of our patient there was associated edema nor focal neither diffuse brain swelling.

Extensive and isolated WM microhemorrhages pattern is also reported in critically ill patients, resulting from long term intubation or ECMO[16] [17]. These findings were recently described in a small number of critically ill patients with COVID-19 [18]. Nevertheless, only a subgroup of our patients required ICU hospitalization.

Stroke associated with a generalized thrombotic predisposition in COVID-19 is of particular interest. Four out of the eight patients had cardiovascular risk factors for stroke including atrial fibrillation. One patient had pulmonary emboli, but still the presence of microthrombi in the distal portions of the pulmonary vascular tree cannot be excluded. COVID-19 is associated with a pro- thrombotic state and highly elevated D-dimer levels, and abnormal coagulation parameters have been shown to be associated with poor outcome [19] I In our cohort we observed similar gross coagulation values, but an increased inflammatory response that could be linked with increased microthrombothic events.

Cerebral microbleeds are usually due to extravasation of red blood cells, and in the context of COVID-19 could be due to endothelial dysfunction related to viral binding to the ACE-2 receptors expressed on endothelial cells. Indeed, a recent report described direct viral infection of the endothelial cell and diffuse endothelial inflammation in multiple organ systems [8] .

Endothelial injury can occur in the smallest blood vessels and can be considered as "microangiopathy". Furthermore, activation of the complement related to the viral infection can determine microthrombi formation. We have also to consider that the term "thrombotic" indicates the presence of blood clots. We 
can speculate that microthrombotic or microangiopatic lesions can occur in other organs besides the lungs, as in the kidneys, the heart, and the brain at the level of the cerebral small vessels, as previously described [20] [21] [22].

In our series the fact that hemorrhage was not evident on gradient echo (GE) sequences (Fig 3) suggests the presence of thrombosis in the vessel walls rather than in the brain parenchyma or in the vessel lumen. SWI sequence can detect micro- and macrohemorhages and delineate cerebral microvasculature and can also reveal low-flow vascular malformations; furthermore, it provides differentiation of calcium from hemorrhage in the brain. Oxyhemoglobin is diamagnetic in nature, whereas deoxyhemoglobin is paramagnetic. The paramagnetic deoxyhemoglobin serves as an intrinsic contrast agent on SWI sequences, and is low in signal. This causes magnetic field inhomogeneity due to two effects: a reduction of T2* and a phase difference between the vessel and its surrounding tissue. This property also forms the basic principle for blood oxygen level dependent functional and venographic imaging. This physical aspect of the SWI sequence can explain why the majority of focal T2 hypointensities are not seen on GE, also supporting the presence not of a true hemorrhage but at least of microthrombosis, that can be located in the vessel wall rather than in the vessel lumen. These findings can be suspicious for endothelial microbleeds (EMBs)/microthrombi.

To support this idea more research on pathology and correlation between MRI and pathologic findings should be performed.

Limitation of this study can be the small sample size; however, to our knowledge, this is the largest series described of patients studied on 3T machine with SWI alterations

In conclusion, we hypothesize that SWI abnormalities in patients with COVID 19 disease with neurological symptoms may correspond to a microvascular endothelial damage with formation of fine thrombi that can be located everywhere in the brain, eventually associated to slow flow in vessels lodged into an area of vascular caliber reduction as in cerebral microvessels, thus configuring a thrombotic microangiopathy. A larger prospective study would be necessary to clarify if this pattern of susceptibility imaging abnormalities as observed in this subset of COVID-19 patients with neurological manifestations, may be related to a thrombotic microangiopathy. In this perspective, histopathological correlation could be enlightening.

\section{Declarations}

\section{1. [Acknowledgements]}

none

\section{Funding}

The authors state that this work has not received any funding.

\section{Compliance with Ethical Standards}

\section{Guarantor:}

The scientific guarantor of this publication is Simonetta Gerevini

\section{Conflict of Interest:}

The authors of this manuscript declare no relationships with any companies, whose products or services may be related to the subject matter of the article.

\section{Statistics and Biometry:}

No complex statistical methods were necessary for this paper furthermore, MC and LGL have statistical expertise

\section{Informed Consent:}

Only if the study is on human subjects:

Written informed consent was waived by the Institutional Review Board.

Only if the study is on animals: N/A

Approval from the institutional animal care committee was not required.

\section{Ethical Approval:}

Institutional Review Board approval was obtained.

Collection and scientific use of the patient's data are part of a larger observational study protocol approved by the hospital ethics committee (reg 2020144). 


\section{Study subjects or cohorts overlap:}

Some study subjects or cohorts have not been previously reported

\section{Methodology}

Methodology:

- retrospective

- observational

- performed at one institution

\section{Abbreviations}

COVID-19: coronavirus disease 2019

SWI: susceptibility weighted imaging

SARS-CoV-2: severe acute respiratory syndrome coronavirus 2

CRP: C-reactive protein

IL-6: interleuchine six

ACE2: angiotensin converting enzyme 2

RT-PCR: real-time reverse-transcriptase polymerase-chain-reaction

BAL: bronchoalveolar lavage

LDH: lactate dehydrogenase

PLT: platelets

PaO2: partial pressure of oxygen

FiO2: fraction of inspired oxygen

Ax : axial

TOF: time-of-flight

Fs: fat suppression

Sag:sagittal

GE: gradient echo sequences

AVG: average

SD: standard deviation

ICUs :Intensive Care Units

NIV: non -invasive methods application for respiratory support

CPAP: Continuous Positive Airway Pressure

N-MRI: Negative MRI

P-MRI: positive MRI

MAS: macrophage activation syndrome

DIC: disseminated intravascular coagulopathy

HACE: high altitude cerebral edema

ECMO: ExtraCorporeal Membrane Oxygenation 
WM: white matter

T2*: T2 star

EMBs: endothelial microbleeds

days H-MR: days passed between hospitalization and MRI

PE: pulmonary embolism

NA: not assessed

SE: splanchnic hemorrhage

Neuro D: neurological disorders

Ex: exitus

Pt:patient

LOBAR MB: lobar microbleeds

MONO: monolateral

BIL: bilateral

CC: corpus callosum

SUBA: subarachnoid hypointensity

LIN: linear hypoitensity

SUP: supratentorial

INF: infratentorial

B-STEM: brainstem

INT CAPS: internal capsule

THAL: thalamus

ME: meningeal enhancement

\section{References}

1. Mao L, Jin H, Wang M, et al (2020) Neurologic Manifestations of Hospitalized Patients with Coronavirus Disease 2019 in Wuhan, China. JAMA Neurol. https://doi.org/10.1001/jamaneurol.2020.1127

2. Varatharaj A, Thomas N, Ellul MA, et al (2020) Neurological and neuropsychiatric complications of COVID-19 in 153 patients: a UK-wide surveillance study. Lancet Psychiatry S221503662030287X. https://doi.org/10.1016/S2215-0366(20)30287-X

3. Paterson RW, Brown RL, Benjamin L, et al (2020) The emerging spectrum of COVID-19 neurology: clinical, radiological and laboratory findings. Brain awaa240. https://doi.org/10.1093/brain/awaa240

4. Verdecchia P, Cavallini C, Spanevello A, Angeli F (2020) The pivotal link between ACE2 deficiency and SARS-CoV-2 infection. Eur J Intern Med 76:1420. https://doi.org/10.1016/j.ejim.2020.04.037

5. Mehta P, McAuley DF, Brown M, et al (2020) COVID-19: consider cytokine storm syndromes and immunosuppression. The Lancet 395:1033-1034. https://doi.org/10.1016/S0140-6736(20)30628-0

6. Chen G, Wu D, Guo W, et al (2020) Clinical and immunological features of severe and moderate coronavirus disease 2019. J Clin Invest 130:26202629. https://doi.org/10.1172/JCl137244

7. Baig AM, Khaleeq A, Ali U, Syeda H (2020) Evidence of the COVID-19 Virus Targeting the CNS: Tissue Distribution, Host-Virus Interaction, and Proposed Neurotropic Mechanisms. ACS Chem Neurosci 11:995-998. https://doi.org/10.1021/acschemneuro.0c00122

8. Varga Z, Flammer AJ, Steiger P, et al (2020) Endothelial cell infection and endotheliitis in COVID-19. The Lancet 395:1417-1418. https://doi.org/10.1016/S0140-6736(20)30937-5

9. Brisse E, Wouters CH, Andrei G, Matthys P (2017) How Viruses Contribute to the Pathogenesis of Hemophagocytic Lymphohistiocytosis. Front Immunol 8:1102. https://doi.org/10.3389/fimmu.2017.01102 
10. Klok FA, Kruip MJHA, van der Meer NJM, et al (2020) Incidence of thrombotic complications in critically ill ICU patients with COVID-19. Thromb Res 191:145-147. https://doi.org/10.1016/j.thromres.2020.04.013

11. Li Y, Xia L (2020) Coronavirus disease 2019 (COVID-19): Role of chest CT in diagnosis and management. Am J Roentgenol 214:1280-1286. https://doi.org/10.2214/AJR.20.22954

12. Needham EJ, Chou SH-Y, Coles AJ, Menon DK (2020) Neurological Implications of COVID-19 Infections. Neurocrit Care. https://doi.org/10.1007/s12028-020-00978-4

13. McGonagle D, O'Donnell JS, Sharif K, et al (2020) Immune mechanisms of pulmonary intravascular coagulopathy in COVID-19 pneumonia. Lancet Rheumatol 2:e437-e445. https://doi.org/10.1016/S2665-9913(20)30121-1

14. Riech S, Kallenberg K, Moerer O, et al (2015) The Pattern of Brain Microhemorrhages After Severe Lung Failure Resembles the One Seen in HighAltitude Cerebral Edema. Crit Care Med 43:

15. Hackett PH, Yarnell PR, Weiland DA, Reynard KB (2019) Acute and evolving MRI of high-altitude cerebral edema: Microbleeds, edema, and pathophysiology. Am J Neuroradiol 40:464-469. https://doi.org/10.3174/ajnr.A5897

16. Fanou EM, Coutinho JM, Shannon P, et al (2017) Critical Illness-Associated Cerebral Microbleeds. Stroke 48:1085-1087. https://doi.org/10.1161/STROKEAHA.116.016289

17. Liebeskind DS, Sanossian N, Sapo ML, Saver JL (2013) Cerebral Microbleeds After Use of Extracorporeal Membrane Oxygenation in Children. J Neuroimaging 23:75-78. https://doi.org/10.1111/j.1552-6569.2012.00723.x

18. Radmanesh A, Derman A, Lui YW, et al (2020) COVID-19 -associated Diffuse Leukoencephalopathy and Microhemorrhages. Radiology 202040. https://doi.org/10.1148/radiol.2020202040

19. Tang N, Li D, Wang X, Sun Z (2020) Abnormal coagulation parameters are associated with poor prognosis in patients with novel coronavirus pneumonia. J Thromb Haemost 18:844-847. https://doi.org/10.1111/jth.14768

20. Magro C, Mulvey JJ, Berlin D, et al (2020) Complement associated microvascular injury and thrombosis in the pathogenesis of severe COVID-19 infection: a report of five cases. Transl Res 1-13. https://doi.org/10.1016/j.trsl.2020.04.007

21. Coolen T, Lolli V, Sadeghi N, et al (2020) Early postmortem brain MRI findings in COVID-19 non-survivors. medRxiv 2020.05.04.20090316. https://doi.org/10.1101/2020.05.04.20090316

22. Noris M, Benigni A, Remuzzi G (2020) The case of complement activation in COVID-19 multiorgan impact. Kidney Int 98:314-322. https://doi.org/10.1016/j.kint.2020.05.013

\section{Tables}

Table 1: Demographic and clinical data 


\begin{tabular}{|c|c|c|c|c|c|c|c|c|c|c|c|c|c|}
\hline Pt & Age & Sex & ICU & Intubation & Days & Neuro D & Comorbidities & $\begin{array}{l}\text { Chest X-Ray } \\
\text { Positivity }\end{array}$ & $\begin{array}{l}\text { Chest CT } \\
\text { Positivity }\end{array}$ & PE & SE & $\begin{array}{l}\text { Days } \\
\text { H-Mr }\end{array}$ & Ex \\
\hline 1 & 75 & $M$ & No & No & 21 & Confusion & $\begin{array}{l}\text { Cardiopathy, } \\
\text { Hypertension, } \\
\text { CV Disease }\end{array}$ & Yes & Yes & NA & NA & 21 & Yes \\
\hline 2 & 62 & $\mathrm{~F}$ & Yes & Yes & 36 & Confusion & Neoplasia & Yes & Yes & Yes & NA & 36 & No \\
\hline 3 & 60 & $M$ & No & No & 3 & Stroke & $\begin{array}{l}\text { Cardiopathy, } \\
\text { Hypertension, } \\
\text { CV Disease, } \\
\text { Obesity }\end{array}$ & Yes & Yes & No & NA & 3 & No \\
\hline 4 & 55 & M & Yes & Yes & 22 & $\begin{array}{l}\text { Confusion, } \\
\text { Hemorrhagic } \\
\text { Stroke, } \\
\text { Peripheral } \\
\text { Neuropathy }\end{array}$ & None & Yes & Yes & No & NA & 22 & No \\
\hline 5 & 75 & M & No & No & 7 & Confusion & Hypertension & No & Yes & NA & NA & 7 & No \\
\hline 6 & 49 & $\mathrm{~F}$ & Yes & Yes & 32 & $\begin{array}{l}\text { Hemorrhagic } \\
\text { Stroke, } \\
\text { Confusion, } \\
\text { Peripheral } \\
\text { Neuropathy }\end{array}$ & None & Yes & Yes & No & NA & 32 & No \\
\hline 7 & 64 & $M$ & Yes & Yes & 42 & Confusion & None & No & Yes & NA & NA & 42 & No \\
\hline 8 & 56 & $M$ & Yes & Yes & 95 & $\begin{array}{l}\text { Movement } \\
\text { Disorders }\end{array}$ & None & Yes & Yes & NA & NA & 95 & No \\
\hline 9 & 51 & M & Yes & Yes & 84 & Confusion & None & Yes & Yes & NA & Yes & 84 & No \\
\hline 10 & 86 & $\mathrm{~F}$ & No & No & 12 & $\begin{array}{l}\text { Epileptic } \\
\text { Seizure }\end{array}$ & $\begin{array}{l}\text { Cardiopathy, } \\
\text { Hypertension, } \\
\text { COPD }\end{array}$ & No & Yes & No & NA & 12 & No \\
\hline
\end{tabular}

Legend: days: days of intubation; days H-MR: days passed between hospitalization and MRI; PE: pulmonary embolism; NA: not assessed; SE: splanchnic hemorrhage; Neuro D: neurological disorders; Ex: exitus.

Table 2: Laboratory and ventilation data analysis 


\begin{tabular}{|c|c|c|c|}
\hline & Positive-MRI & Negative-MRI & $P$ value \\
\hline & Median value $[25-75 \%]$ & Median value $[25-75 \%]$ & \\
\hline Leukocytes $10^{9} / \mathrm{lt}$ & $13[12.5-18.5]$ & $11[9-15]$ & 0.14 \\
\hline Lymphocytes $10^{9} / \mathrm{lt}$ & $0.5[0.3-0.7]$ & $0.7[0.5-1.4]$ & 0.03 \\
\hline PLTmax $10^{9} / \mathrm{lt}$ & 334 [262.5-457.5] & 308 [265-475.3] & 0.81 \\
\hline PLTmin $10^{9} / \mathrm{lt}$ & 121 [97-170] & 165 [110.8-217.8] & 0.21 \\
\hline CRP mg/dL & 34 [22-38] & 11 [3.6-28] & 0.002 \\
\hline$\underline{\mathrm{LL}-6} \mathrm{pg} / \mathrm{ml}$ & 271 [168-1012] & 29 [6.8-297.5] & 0.03 \\
\hline LDHU/I & 583 [417-707.5] & 366.5 [259.5-612] & 0.09 \\
\hline Fibrinogen $\mathrm{mg} / \mathrm{dL}$ & 902 [855-1067] & 637 [413.5-836.5] & 0.004 \\
\hline D -Dimer ng/mL & 4895 [1049-11247] & 2463 [844-6048] & 0.36 \\
\hline $\mathrm{PaO} 2 \mathrm{~min} \mathrm{mmHg}$ & $67[56-78.5]$ & $68[58-71.5]$ & 0.98 \\
\hline $\mathrm{FiO} \%$ & 75 [65-85] & $65[60-80]$ & 0.33 \\
\hline $\mathrm{PaO2} / \mathrm{FiO} 2$ & 100 [95-109.5] & 100 [83.5-101.5] & 0.59 \\
\hline
\end{tabular}

Legend: PLT: platelets; CRP: C-reactive protein; IL-6: interleuchine-6; LDH: lactate dehydrogenase; Pa02: partial pressure of oxygen; FiO2: fraction of inspired oxygen

Table 3: MRI data

MR microbleeds data

\begin{tabular}{|c|c|c|c|c|c|c|c|c|c|c|c|c|c|c|c|c|c|}
\hline $\mathrm{Pt}$ & $\begin{array}{l}\text { LOBAR } \\
\text { MB }\end{array}$ & LOBE & MONO & BIL & $\mathrm{CC}$ & splenium & genu & corpus & SUBA & LIN & DOT & SUP & INF & $\begin{array}{l}\text { B- } \\
\text { STEM }\end{array}$ & $\begin{array}{l}\text { INT } \\
\text { CAPS }\end{array}$ & THAL & $\mathrm{ME}$ \\
\hline 1 & $x$ & $\mathrm{O}, \mathrm{T}, \mathrm{F}$ & & $x$ & $x$ & $x$ & & $x$ & $x$ & & $x$ & $x$ & $x$ & $x$ & & $x$ & $x$ \\
\hline 2 & $x$ & F,T,O,P,I & & $x$ & $x$ & $x$ & $x$ & $x$ & $x$ & $x$ & $x$ & $x$ & $x$ & $x$ & $x$ & $x$ & \\
\hline 3 & $x$ & $\mathrm{~T}$ & $x$ & & $x$ & & $x$ & & & & $x$ & $x$ & & & & & \\
\hline 4 & $x$ & NO & & $x$ & $x$ & $x$ & & & $x$ & & $x$ & $x$ & $x$ & & $x$ & & \\
\hline 5 & $x$ & $\mathrm{~T}, \mathrm{O}$ & & $x$ & $x$ & $x$ & & $x$ & $x$ & & $x$ & $x$ & $x$ & $x$ & & $x$ & $x$ \\
\hline 6 & $\mathrm{x}$ & $\mathrm{T}, \mathrm{O}, \mathrm{F}$ & & $x$ & $\mathrm{x}$ & $\mathrm{x}$ & $\mathrm{x}$ & $\mathrm{x}$ & $\mathrm{x}$ & $x$ & $x$ & $x$ & $x$ & $x$ & $\mathrm{x}$ & & \\
\hline 7 & & & $x$ & & $x$ & & $x$ & & & & $x$ & $x$ & & & & & $x$ \\
\hline 8 & $x$ & $\mathrm{~F}, \mathrm{~T}, \mathrm{O}, \mathrm{P}$ & & $x$ & $x$ & $x$ & $x$ & $x$ & $x$ & $x$ & $x$ & $x$ & $x$ & $x$ & $x$ & $x$ & $x$ \\
\hline 9 & $x$ & $\mathrm{~T}, \mathrm{O}, \mathrm{F}, \mathrm{P}$ & & $x$ & $x$ & $x$ & $x$ & $x$ & & & $x$ & $x$ & & & & & \\
\hline 10 & $x$ & O,F,P & & $x$ & $x$ & $x$ & & & & & $x$ & $x$ & $x$ & & & & \\
\hline
\end{tabular}

Pt:patient; LOBAR MB: lobar microbleeds; MONO: monolaterale; BIL: bilateral; CC: corpus callosum SUBA: subarachnoid hypointensity; LIN: linear hypoitensity; SUP: supratentorial; INF: infratentorial; B-STEM: brainstem; INT CAPS: internal capsule, THAL: thalamus; ME: meningeal enhancement

\section{Figures}




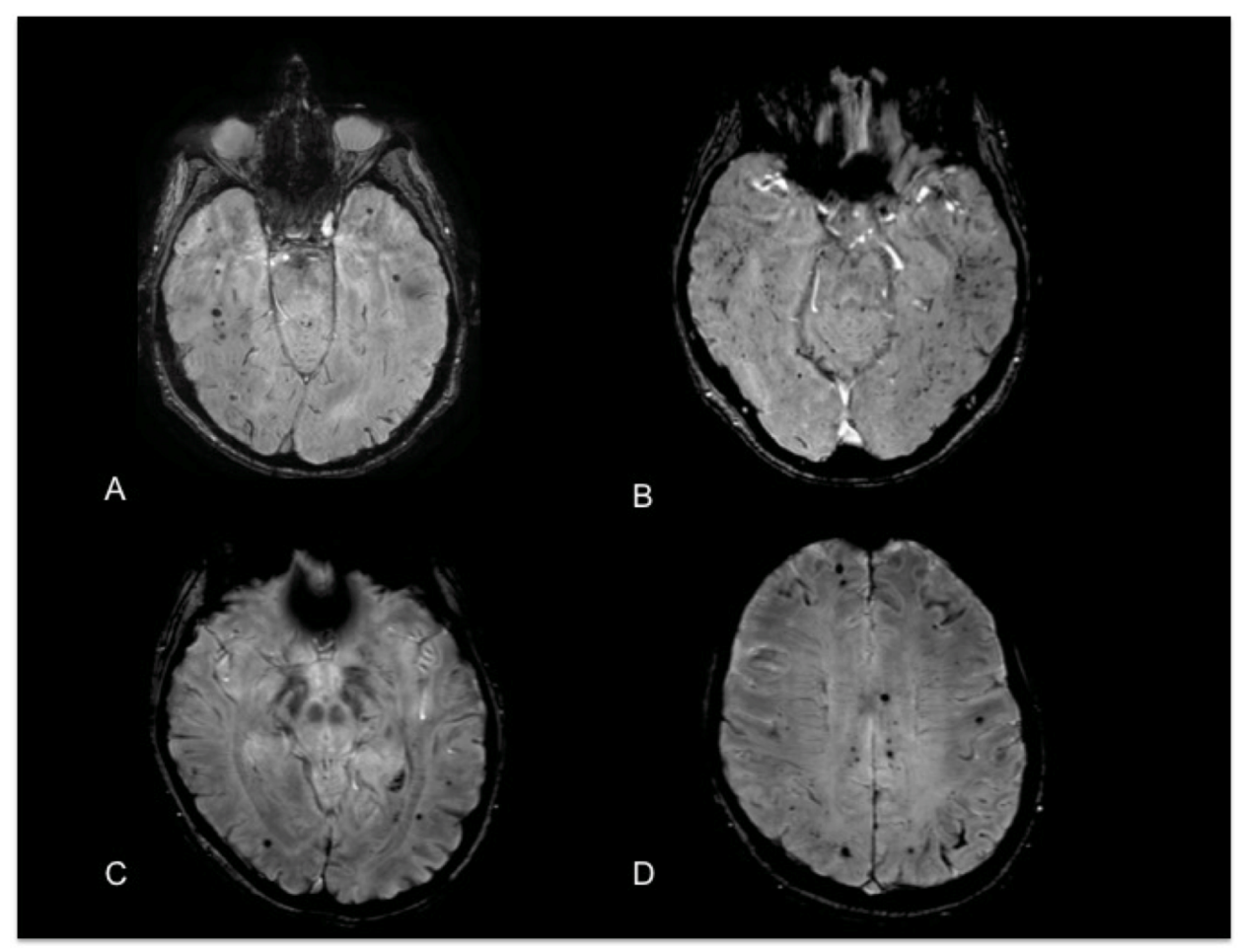

\section{Figure 1}

Axial SWI images showing numerous punctate and linear susceptibility abnormalities in the white matter mainly located at the juxtacortical junction in the temporal lobes (panel A pt. N. 5, B pt. N. 2) and more diffuse in the frontal, occipital and temporal lobes (panel C and D, pt. N. 6). 


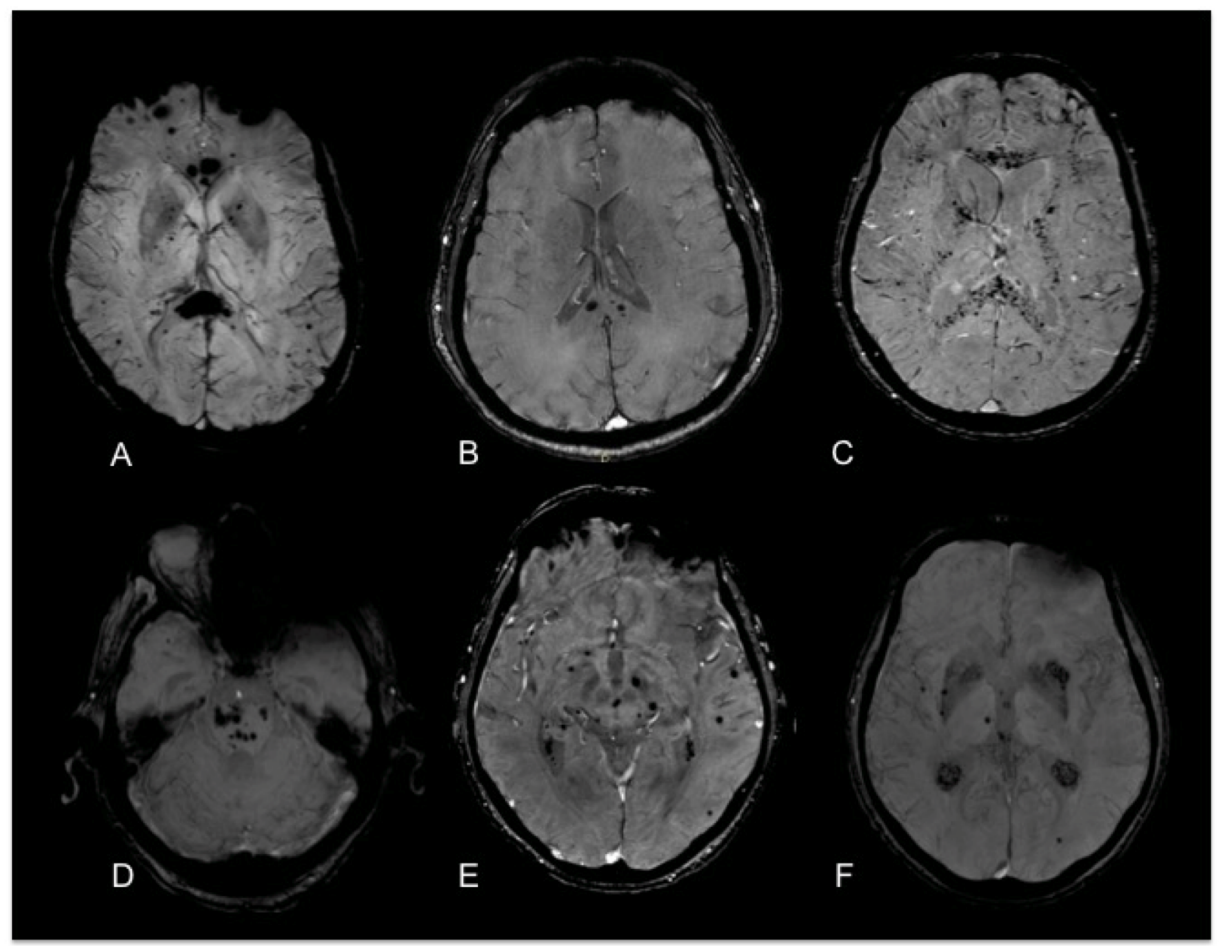

\section{Figure 2}

Axial SWI images showing multiple hypointensities mainly located in the splenium and genu of the corpus callosum (A pt. N. 6 and B pt N.4) and at the level of internal capsule (C, pt. N.2), somehow depicting the perivascular spaces. SWI images at posterior fossa and the level of basal cisterns show diffuse micro hemorrhagic/thrombotic lesions predominantly involving the brainstem ( $D$ and E, pt. N.1). Similar hypointense foci are also present in the thalami (F, pt. N.1). 


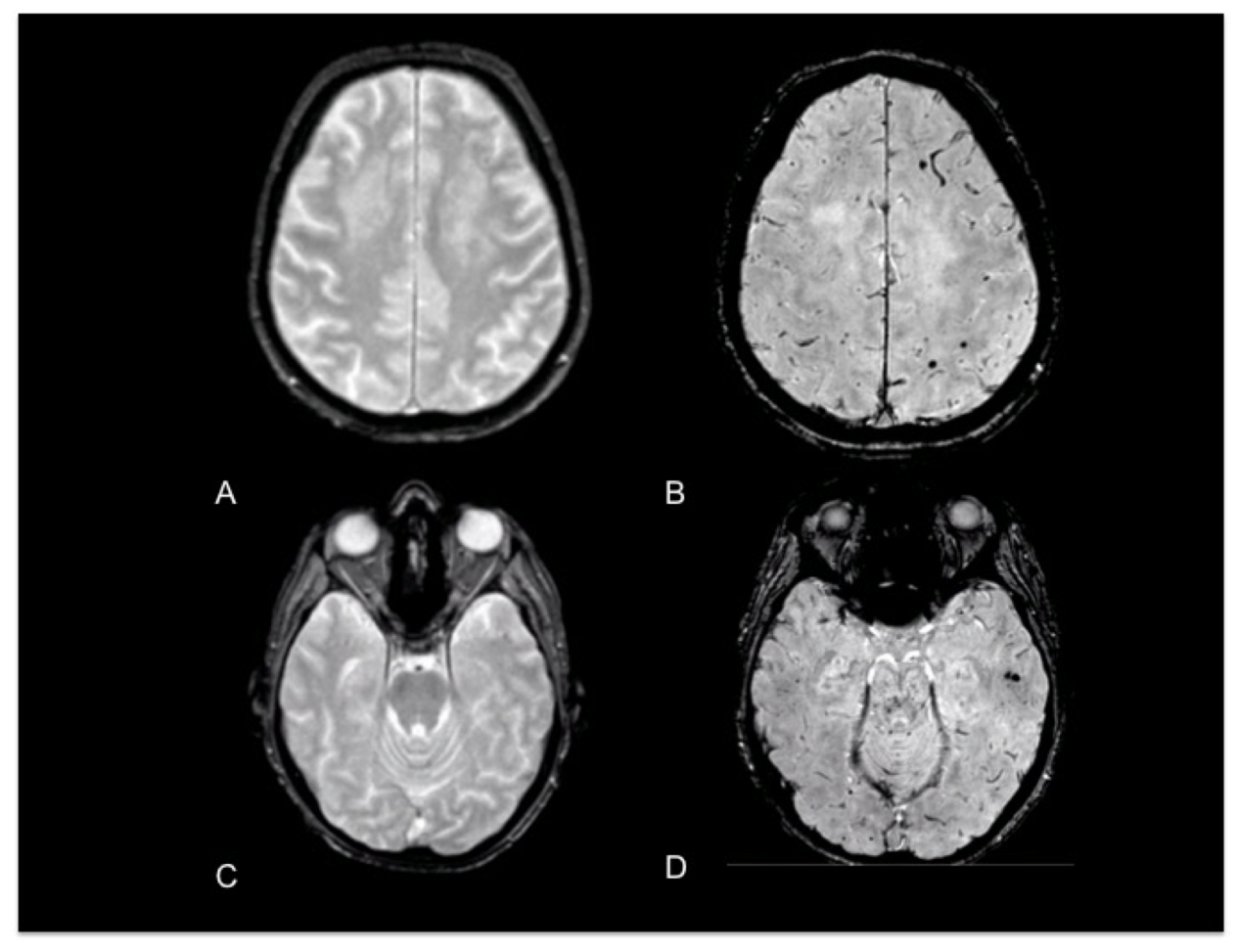

\section{Figure 3}

Comparison between images acquired by GE (A and C) and SWI brain MRI sequences (B and D), showing higher diagnostic performance of SWI in the detection of microthrombotic / microhemorrhagic hypointense lesions. Brain MR images refer to patient $\mathrm{n} .8$. 